\title{
$G$-equivalence in group algebras and minimal abelian codes
}

\author{
Raul Antonio Ferraz, Marinês Guerreiro, and César Polcino Milies,
}

\begin{abstract}
Let $G$ be a finite abelian group and $\mathbb{F}$ a field such that $\operatorname{char}(\mathbb{F}) \backslash|G|$. Denote by $\mathbb{F} G$ the group algebra of $G$ over $\mathbb{F}$. A (semisimple) abelian code is an ideal of $\mathbb{F} G$. Two codes $\mathcal{I}_{1}$ and $\mathcal{I}_{2}$ of $\mathbb{F} G$ are $G$-equivalent if there exists an automorphism $\psi$ of $G$ whose linear extension to $\mathbb{F} G$ maps $\mathcal{I}_{1}$ onto $\mathcal{I}_{2}$.

In this paper we give a necessary and sufficient condition for minimal abelian codes to be $G$-equivalent and show how to correct some results in the literature.
\end{abstract}

Index Terms-group algebra, $G$-equivalence, primitive idempotent, abelian codes.

\section{INTRODUCTION}

$\mathbf{L}$ ET $G$ be a finite group and $\mathbb{F}$ a finite field such that $\operatorname{char}(\mathbb{F}) \quad \backslash \quad|G|$. Two ideals $\mathcal{I}_{1}$ and $\mathcal{I}_{2}$ of the group algebra $\mathbb{F} G$ are said to be G-equivalent if there exists an automorphism $\psi$ of $G$ whose linear extension to $\mathbb{F} G$ maps $\mathcal{I}_{1}$ onto $\mathcal{I}_{2}$.

This definition was introduced R.L. Miller [9] in the context of Coding Theory. S.D. Berman [1] and, independently, F.J. MacWilliams [8] defined abelian codes as ideals in finite abelian group algebras and R.L. Miller [9] used $G$-equivalence to compare codes with the same weight distribution.

In this paper, we address the problem of determining $G$ equivalence of minimal ideals in semisimple abelian group algebras and prove that the $G$-equivalence classes of minimal ideals depend on the structure of the lattice of the subgroups of $G$.

In Section $\Pi$ we prove preliminary results about idempotents and in Section III we establish a correspondence between $G$-equivalence classes of minimal abelian ideals in $\mathbb{F} G$ and certain isomorphism classes of subgroups of $G$. In Section IV we use these facts to show that some of the results of [9] are not correct and, in the final section, we exhibit particular cases for which such results hold.

\section{SUbGRoups AND IDEMPOTENTS}

The irreducible central idempotents of the rational group algebra $\mathbb{Q} G$ were computed in [5, Theorem 1.4] in the case when $G$ is abelian; in [7, Theorem 2.1] when $G$ is nilpotent; in [10, Theorem 4.4] when $G$ is abelian-by-supersolvable

R.A. Ferraz and C. Polcino Milies are with the Instituto de Matemática e Estatística, Universidade de São Paulo, Caixa Postal 66281, CEP 05314-970 - São Paulo - SP (Brasil), supported by CNPq, Proc. 300243/79-0(RN) and FAPESP, Proc. 09/52665-0. E-mail: raul@ime.usp.br and polcino@ime.usp.br M. Guerreiro is with Departamento de Matemática, Universidade Federal de Viçosa, CEP 36570-000 - Viçosa-MG (Brasil), supported by CAPES, PROCAD 915/2010 and FAPEMIG, APQ CEX 00438-08. E-mail: marines @ufv.br

This work was presented in part at the 2011 IEEE Information Theory Workshop (ITW), Paraty-RJ (Brasil) 16-20 October, 2011.

Manuscript received ???, 20012; revised ???, 2012. and in [2, Theorem 7] an algorithm to write the primitive idempotents in given.

In what follows, we shall establish a correspondence between primitive idempotents of $\mathbb{F} G$ and certain subgroups of $G$.

Definition II.1. Let $G$ be a group. A subgroup $H$ of $G$ is said a co-cyclic subgroup if the factor group $G / H \neq 1$ is cyclic.

We use the notation

$$
\mathcal{S}_{c c}(G)=\{H \mid H \text { is a co-cyclic subgroup of } G\} .
$$

We recall the following results that are used throughout this paper.

Let $G$ be a finite abelian $p$-group and $\mathbb{F}$ a field such that $\operatorname{char}(\mathbb{F}) \times|G|$. Given a subgroup $H$ of $G$, denote $\widehat{H}=\frac{1}{|H|} \sum_{h \in H} h$ and, for an element $x \in G$, set $\widehat{x}=\widehat{\langle x\rangle}$. For each co-cyclic subgroup $H$ of $G$, we can construct an idempotent of $\mathbb{F} G$. In fact, we remark that, since $G / H$ is a cyclic $p$-group, there exists a unique subgroup $H^{\sharp}$ of $G$ containing $H$ such that $\left|H^{\sharp} / H\right|=p$. Then $e_{H}=\widehat{H}-\widehat{H^{\sharp}}$ is an idempotent and we consider the set

$$
\{\widehat{G}\} \cup\left\{e_{H}=\widehat{H}-\widehat{H^{\sharp}} \mid H \in \mathcal{S}_{c c}(G), G / H \neq\{1\}\right\} .
$$

In the case of a rational abelian group algebra $\mathbb{Q} G$, the set above is the set of primitive central idempotents [5. Theorem 1.4]. Also, the following results holds.

Theorem II.2. [3, Lemma 5] Let $p$ be a prime rational integer and $G$ a finite abelian group of exponent $p^{n}$ and $\mathbb{F}_{q}$ a finite field such that $p \nmid q$. Then (1) is a set of pairwise orthogonal idempotents of $\mathbb{F}_{q} G$ whose sum is equal to 1 .

Theorem II.3. [3, Theorem 4.1] Under the hypotheses above, the set (1) is the set of primitive idempotents of $\mathbb{F}_{q} G$ if and only if $o(\bar{q})=\phi\left(p^{n}\right)$ in $U\left(\mathbb{Z}_{p^{n}}\right)$, where $\varphi$ denotes Euler's totient function.

We shall repeatedly use the following rather obvious fact.

Lemma II.4. Let $G$ be a finite abelian $p$-group and $H \leq G$. Then $G / H$ is a cyclic group if and only if there exists a unique subgroup $L$ such that $H<L \leq G$ and $[L: H]=p$.

In the sequel, for a finite abelian group $G$, we write $G=$ $G_{p_{1}} \times \cdots \times G_{p_{t}}$, where $G_{p_{i}}$ denotes the $p_{i}$-Sylow subgroup of $G$, for the distinct positive prime numbers $p_{1}, \ldots, p_{t}$.

Lemma II.5. Let $G=G_{p_{1}} \times \cdots \times G_{p_{t}}$ be a finite abelian group and $H \in \mathcal{S}_{c c}(G)$. Write $H=H_{p_{1}} \times \cdots \times H_{p_{t}}$, where $H_{p_{i}}$ is the $p_{i}$-Sylow subgroup of $H$. Then each subgroup $H_{p_{i}}$ is co-cyclic in $G_{p_{i}}, 1 \leq i \leq t$. 
Proof: For $H \in \mathcal{S}_{c c}(G)$, the quotient $G / H \cong G_{p_{1}} / H_{p_{1}} \times$ $\cdots \times G_{p_{t}} / H_{p_{t}}$ is cyclic, hence each factor $G_{p_{i}} / H_{p_{i}}$ must be cyclic. Therefore, $H_{p_{i}} \in \mathcal{S}_{c c}\left(G_{p_{i}}\right), 1 \leq i \leq t$.

With the notation above, for each $H \in \mathcal{S}_{c c}(G)$, define an idempotent $e_{H} \in \mathbb{F} G$ as follows. For each $1 \leq i \leq t$, either $H_{p_{i}}=G_{p_{i}}$ or there exists a unique subgroup $H_{p_{i}}^{\sharp}$ such that $\left[H_{p_{i}}^{\sharp}: H_{p_{i}}\right]=p_{i}$. Thus, let $e_{H_{p_{i}}}=\widehat{G_{p_{i}}}$ or $e_{H_{p_{i}}}=\widehat{H_{p_{i}}}-\widehat{H_{p_{i}}^{\sharp}}$, respectively, and define

$$
e_{H}=e_{H_{p_{1}}} e_{H_{p_{2}}} \cdots e_{H_{p_{t}}} .
$$

For any other $K \in \mathcal{S}_{c c}(G)$, with $K \neq H$, we have $K_{p_{i}} \neq$ $H_{p_{i}}$, for some $1 \leq i \leq t$, hence $e_{H_{p_{i}}} e_{K_{p_{i}}}=0$ and so $e_{H} e_{K}=$ 0 . Thus, we have the following.

Proposition II.6. Let $G$ be a finite abelian group and $\mathbb{F} a$ field such that $\operatorname{char}(\mathbb{F}) \backslash|G|$. Then

$$
\mathcal{B}=\left\{e_{H} \mid H \in \mathcal{S}_{c c}(G)\right\}
$$

is a set of orthogonal idempotents of $\mathbb{F} G$.

A similar construction of idempotents for rational group algebras of abelian groups is given in [5, Section VII.1]. For the rational case, these idempotents are primitive while for finite fields this is usually not true.

To study the $G$-equivalence of ideals, we need to understand how the group of automorphisms $\operatorname{Aut}(G)$ acts on the lattice of the subgroups of $G$ and hence on the idempotents in the group algebra which arise from these subgroups. From now on, we use the same notation for an automorphism of the group $G$ and its linear extension to the group algebra $\mathbb{F} G$.

Lemma II.7. Let $G$ be a finite abelian group, $H \in \mathcal{S}_{c c}(G)$ and $e_{H}$ its corresponding idempotent defined as in (2). Then, for any $\psi \in \operatorname{Aut}(G)$, we have $\psi\left(e_{H}\right)=e_{\psi(H)}$.

Proof: By Lemma $I .5=H_{p_{1}} \times H_{p_{2}} \times \cdots \times H_{p_{t}}$, where $H_{p_{i}}$ is the $p_{i}$-Sylow subgroup of $H$ which is co-cyclic in $G_{p_{i}}$ (the $p_{i}$-Sylow subgroup of $G$ ), for each $1 \leq i \leq t$. Since $\psi \in \operatorname{Aut}(G), \psi(H)=\psi\left(H_{p_{1}}\right) \times \psi\left(H_{p_{2}}\right) \times \cdots \times \psi\left(H_{p_{t}}\right)$. Then each $\psi\left(H_{p_{i}}\right)$ is the $p_{i}$-Sylow subgroup of $\psi(H)$ and is also co-cyclic in $G_{p_{i}}$. Hence $\psi\left(H_{p_{i}}^{\sharp}\right)=\psi(H)_{p_{i}}^{\sharp}$ and the result follows.

Lemma II.8. Let $G$ be a finite abelian group and $\mathbb{F}$ a field such that $\operatorname{char}(\mathbb{F}) \backslash|G|$. Then, in the group algebra $\mathbb{F} G$, we have:

$$
1=\widehat{G}+\sum_{H \in \mathcal{S}_{c c}(G)} e_{H}
$$

Proof: Let $G=G_{p_{1}} \times G_{p_{2}} \times \cdots \times G_{p_{t}}$, with $G_{p_{i}}$ the $p_{i}$-Sylow subgroup of $G, 1 \leq i \leq t$. By Theorem II.2 we have

$$
1=\widehat{G}_{p_{i}}+\sum_{H_{p_{i}} \in \mathcal{S}_{c c}\left(G_{p_{i}}\right)}\left(\widehat{H_{p_{i}}}-\widehat{H_{p_{i}}^{\sharp}}\right) .
$$

Thus:

$$
\begin{aligned}
1 & =\prod_{i=1}^{t}\left(\widehat{G}_{p_{i}}+\sum_{H_{p_{i}} \in \mathcal{S}_{c c}\left(G_{p_{i}}\right)}\left(\widehat{H_{p_{i}}}-\widehat{H_{p_{i}}^{\sharp}}\right)\right) \\
& =\sum e_{H_{p_{1}}} e_{H_{p_{2}}} \cdots e_{H_{p_{t}}}
\end{aligned}
$$

where either $e_{H_{p_{i}}}=\widehat{G_{p_{i}}}$ or $e_{H_{p_{i}}}=\widehat{H_{p_{i}}}-\widehat{H_{p_{i}}^{\sharp}}, 1 \leq i \leq t$. Therefore,

$$
1=\widehat{G}+\sum_{H \in \mathcal{S}_{c c}(G)} e_{H}
$$

Lemma II.9. Let $G$ be a finite abelian group and $\mathbb{F}$ a field such that $\operatorname{char}(\mathbb{F}) \backslash|G|$. For each primitive idempotent $e \in$ $\mathbb{F} G$, there exists a unique $H \in \mathcal{S}_{c c}(G)$ such that $e \cdot e_{H}=e$ and $e \cdot e_{K}=0$, for any other $K \in \mathcal{S}_{c c}(G)$.

Proof: By Lemma I.8 $1=\widehat{G}+\sum_{H \in \mathcal{S}_{c c}(G)} e_{H}$. Multiplying by $e$, we have:

$$
e=e\left(\widehat{G}+\sum_{H \in \mathcal{S}_{c c}(G)} e_{H}\right)=e \cdot \widehat{G}+\sum_{H \in \mathcal{S}_{c c}(G)} e \cdot e_{H}
$$

As $e_{H} \cdot e_{K}=0$, for $H \neq K$ co-cyclic subgroups of $G$, the right hand side of (5) is a sum of orthogonal idempotents. Therefore, as $e$ is a primitive idempotent, only one summand is non-zero and this proves the lemma.

Set:

$\mathcal{P}(\mathbb{F} G)=\{e \in \mathbb{F} G \mid e$ is a primitive idempotent in $\mathbb{F} G\}$.

Under the same hypotheses of Lemma II.9 the following map is well-defined:

$$
\begin{array}{clc}
\Phi: \mathcal{P}(\mathbb{F} G) & \longrightarrow & \mathcal{S}_{c c}(G) \\
e & \longmapsto & \Phi(e)=H_{e},
\end{array}
$$

where $H_{e}$ is the unique co-cyclic subgroup of $G$ such that $e \cdot e_{H_{e}}=e$.

Theorem II.10. Let $G$ be a finite abelian group, $\mathbb{F}$ a field such that $\operatorname{char}(\mathbb{F}) \backslash|G|$ and $H \in \mathcal{S}_{c c}(G)$. Then $e_{H}$ is the sum of all primitive idempotents $e \in \mathcal{P}(\mathbb{F} G)$ such that $\Phi(e)=H$.

$$
\begin{aligned}
& \text { Proof: Write } 1=\sum_{e \in \mathcal{P}(\mathbb{F} G)} e . \text { Then } \\
& e_{H}=\sum_{e \in \mathcal{P}(\mathbb{F} G)} e_{H} e=\sum_{\Phi(e) \neq H} e_{H} e+\sum_{\Phi(e)=H} e_{H} e=\sum_{\Phi(e)=H} e .
\end{aligned}
$$

Remark II.11. For a primitive idempotent $e \in \mathbb{F} G$ and its correspondent subgroup $H=H_{e}$, as in (6), we have:

$$
e \widehat{K}=e \Leftrightarrow K \leq H \text { and } e \widehat{K}=0 \Leftrightarrow K \not \leq H .
$$

Proof: By (2), $e_{H}=e_{H_{1}} e_{H_{2}} \cdots e_{H_{t}}$, where either $e_{H_{i}}=$ $\widehat{G_{i}}$ or $e_{H_{i}}=\widehat{H_{i}}-\widehat{H_{i}^{\sharp}}$, where $H_{i}$ denotes the $p_{i}$-Sylow of $H$, $1 \leq i \leq t$.

Let $K \leq H$. Then $K_{i} \leq H_{i} \leq H_{i}^{\sharp}$, where $K_{i}$ is the $p_{i^{-}}$ Sylow of $K$. Hence $\widehat{K_{i}} \widehat{H_{i}}=\widehat{H_{i}}$ and $\widehat{K_{i}} \widehat{H_{i}^{\sharp}}=\widehat{H_{i}^{\sharp}}$. Thus either $\widehat{K_{i}} \widehat{G_{i}}=\widehat{G_{i}}$ or $\widehat{K}_{i} e_{H_{i}}=\widehat{K_{i}}\left(\widehat{H_{i}}-\widehat{H_{i}^{\sharp}}\right)=\widehat{H_{i}}-\widehat{H_{i}^{\sharp}}=e_{H_{i}}$. Therefore,

$e \widehat{K}=e e_{H} \widehat{K}=e\left(e_{H_{1}} e_{H_{2}} \cdots e_{H_{t}}\right)\left(\widehat{K_{1}} \widehat{K_{2}} \cdots \widehat{K_{t}}\right)=e e_{H}=e$.

If $K \not \leq H$, then there exists at least one $K_{i} \not \leq H_{i}$. In this case, $H_{i}^{\sharp} \subset K_{i} H_{i}$, hence $\widehat{K_{i}} \widehat{H_{i}^{\sharp}}=\widehat{K_{i}} \widehat{H}_{i}$ and $\widehat{K}_{i} e_{H_{i}}=\widehat{K_{i}}\left(\widehat{H_{i}}-\right.$ $\left.\widehat{H_{i}^{\sharp}}\right)=\widehat{K}_{i} \widehat{H}_{i}-\widehat{K}_{i} \widehat{H}_{i}=0$. This proves the remark. 
Proposition II.12. Let $G$ be a finite abelian group and $\mathbb{F}$ a field such that $\operatorname{char}(\mathbb{F}) \backslash|G|$. If $e \in \mathbb{F} G$ is a primitive idempotent, then $G e \cong G / H_{e}$.

Proof: It is clear that $G e$ is a group. Consider $\pi: G \longrightarrow$ Ge given by $\pi(g)=g e$. Clearly $H_{e} \subset \operatorname{ker}(\pi)$. For $g \in$ $\operatorname{ker}(\pi), \pi(g)=e$ implies $g e=e$, hence $\widehat{\langle g\rangle} e=e$ and, by Remark II.11 $\langle g\rangle \subset H_{e}$. Therefore, $g \in H_{e}$ and $\operatorname{ker}(\pi)=$ $H_{e}$, proving the lemma.

Let $G$ be a finite abelian group. We recall that its character group is $G^{*}=\operatorname{Hom}\left(G, \mathbb{C}^{*}\right)$, with multiplication defined by $(f \cdot g)(x)=f(x) \cdot g(x)$, for all $f, g \in G^{*}$ and $x \in G$. Also for a subgroup $H$ of $G$, define

$$
H^{\perp}=\left\{f \in G^{*} \mid f(h)=1, \text { for all } h \in H\right\} .
$$

The following facts on character groups can be found in [12, Chapter 10] and will be used in the next sections.

Theorem II.13. Let $G$ be a finite abelian group. Then:

(1) $G^{*} \cong G$.

(2) If $H \leq G$, then $G$ contains a subgroup isomorphic to $G / H$.

(3) $H^{\perp}$ is a subgroup of $G^{*}$ and $H^{\perp} \cong(G / H)^{*}$.

(4) Let $\mathcal{S}(G)$ be the set of all subgroups of a group $G$. Then the map

$$
\begin{aligned}
\Psi: \mathcal{S}(G) & \longrightarrow \mathcal{S}\left(G^{*}\right) \\
H & \longmapsto H^{\perp}
\end{aligned}
$$

is a bijection and satisfies:

(a) If $H \subset K$ then $H^{\perp} \supset K^{\perp}$ (with proper inclusion preserved).

(b) If $H$ is a cyclic subgroup of order $p^{s}$ in a finite abelian p-group $G$ of exponent $p^{r}$, with $r \geq s$, then $H^{\perp}$ is a co-cyclic subgroup of $G$ such that $G^{*} / H^{\perp} \cong C_{p^{s}} \cong H$.

(c) If $H$ is a co-cyclic subgroup such that $G / H \cong C_{p^{s}}$ in a finite abelian p-group $G$ of exponent $p^{r}$, with $r \geq s$, then $H^{\perp}$ is a cyclic subgroup isomorphic to $C_{p^{s}}$.

\section{III. $G$-ISOMORPHISMS OF SUBGROUPS}

We say that two subgroups $H$ and $K$ of a group $G$ are $G$-isomorphic if there exists an automorphism $\psi \in \operatorname{Aut}(G)$ such that $\psi(H)=K$.

Notice that isomorphic subgroups are not necessarily $G$ isomorphic. For example, for a prime $p$, if $G=\langle a\rangle \times\langle b\rangle$ with $o(a)=p^{2}$ and $o(b)=p$, then $\left\langle a^{p}\right\rangle$ and $\langle b\rangle$ are isomorphic but not $G$-isomorphic, since $\langle b\rangle$ is contained properly only in $\left\langle a^{p}\right\rangle \times\langle b\rangle$ and $\left\langle a^{p}\right\rangle$ is contained in $\langle a\rangle$ and in $\left\langle a^{i} b\right\rangle$, for all $1 \leq i \leq p-1$.

For finite abelian groups, Propositions III.1, ПII.6 and ЩII.7 below establish a correspondence between $G$-equivalent minimal ideals and $G$-isomorphic subgroups.

Proposition III.1. Let $G$ be a finite abelian group and $\mathbb{F} a$ field such that $\operatorname{char}(\mathbb{F}) \backslash|G|$. If $e, e_{1} \in \mathcal{P}(\mathbb{F} G)$ are such that $\psi(e)=e_{1}$, for some automorphism $\psi \in \operatorname{Aut}(G)$ linearly extended to $\mathbb{F} G$, then

$$
\psi\left(H_{e}\right)=H_{\psi(e)}=H_{e_{1}},
$$

i.e., $H_{e}$ and $H_{e_{1}}$ are $G$-isomorphic.
Proof: Given $e, e_{1} \in \mathcal{P}(\mathbb{F} G)$, from Lemma [II.9, there exist $H_{e}, H_{e_{1}} \in \mathcal{S}_{c c}(G)$ such that

$$
e \cdot e_{H_{e}}=e \quad \text { and } \quad e_{1} \cdot e_{H_{e_{1}}}=e_{1} .
$$

Hence, by Lemma $\llbracket .7$

$$
\psi(e)=\psi\left(e \cdot e_{H_{e}}\right)=\psi(e) \psi\left(e_{H_{e}}\right)=\psi(e) \cdot e_{\psi\left(H_{e}\right)} .
$$

Since $\psi(e)$ is also a primitive idempotent in $\mathbb{F} G$, Lemma II.9 shows that there exists a unique subgroup in $G$ satisfying (7), hence $\psi\left(H_{e}\right)=H_{\psi(e)}$. As $\psi(e)=e_{1}$, we have $\psi\left(H_{e}\right)=H_{e_{1}}$.

The converse of the Proposition III.1 is also true and it will be proved in Proposition $11 \mathrm{II}$.7 as we still need more information. We set

$$
\mathcal{L} \operatorname{Aut}(G)=\{\psi \in \operatorname{Aut}(G) \mid \psi(H)=H, \text { for all } H \leq G\} .
$$

Lemma III.2. Let $G$ be a finite abelian group, $g \in G$ and $r \in \mathbb{N}$ with $\operatorname{gcd}(r, o(g))=1$. Then there exists $\psi \in \mathcal{L} \operatorname{Aut}(G)$ such that $\psi(g)=g^{r}$.

Proof: We can write $|G|=k \cdot s$ such that $k$ and $o(g)$ have the same prime divisors and $\operatorname{gcd}(s, o(g))=\operatorname{gcd}(k, s)=$ 1 . By the Chinese Remainder Theorem, there exists $x \in \mathbb{Z}$ satisfying

$$
x \equiv r(\bmod k) \quad \text { and } \quad x \equiv 1(\bmod s) .
$$

This implies $\operatorname{gcd}(x, k)=\operatorname{gcd}(x, s)=1$, hence $\operatorname{gcd}(x,|G|)=$ 1. Therefore, defining $\psi: G \longrightarrow G$ by $\psi(h)=h^{x}$, we have $\psi \in \mathcal{L} \operatorname{Aut}(G)$ and $\psi(g)=g^{x}=g^{r}$.

Lemma III.3. Let $G$ be a finite abelian group and $\psi \in$ $\operatorname{Aut}(G)$. Then $\psi \in \mathcal{L} \operatorname{Aut}(G)$ if and only if there exists $r \in \mathbb{N}$ such that $\operatorname{gcd}(r,|G|)=1$ and $\psi(g)=g^{r}$, for all $g \in G$.

Proof: Suppose $\psi \in \mathcal{L} \operatorname{Aut}(G)$. For each $g \in G$, we have $\psi(\langle g\rangle)=\langle g\rangle$, hence $\psi(g)=g^{r_{g}}$, for some $r_{g} \in \mathbb{N}$ such that $\operatorname{gcd}\left(r_{g},|G|\right)=1$.

Write $G \cong C_{s_{1}} \times C_{s_{2}} \times \cdots \times C_{s_{t}}$, with $C_{s_{j}}=\left\langle a_{j}\right\rangle$ of order $s_{j}|| G \mid$ and $s_{j+1} \mid s_{j}, 1 \leq j \leq t$. Thus $\psi\left(a_{j}\right)=a_{j}^{r_{j}}$, with $\operatorname{gcd}\left(r_{j},|G|\right)=1,1 \leq j \leq t$. As $s_{j} \mid s_{1}$, then $r_{j} \equiv r_{1}($ $\left.\bmod s_{j}\right), 1 \leq j \leq t$, and $r_{g} \equiv r_{1}\left(\bmod s_{j}\right), 1 \leq j \leq t$. Therefore, we can take $r_{g}=r_{1}$. This proves the lemma, since the converse is clear.

Lemma III.4. Let $G$ be a finite abelian group and $\mathbb{F}$ a field such that $\operatorname{char}(\mathbb{F}) \backslash|G|$. Then $\mathcal{B}=\left\{e_{H} \mid H \in \mathcal{S}_{c c}(G)\right\}$ is both a basis for the algebra

$$
\mathcal{A}=\{\alpha \in \mathbb{F} G \mid \psi(\alpha)=\alpha, \text { for all } \psi \in \mathcal{L} \operatorname{Aut}(G)\}
$$

and the set of primitive idempotents of $\mathcal{A}$.

Proof: For an element $g \in G$, set

$$
\gamma_{g}=\sum_{\substack{\operatorname{gcd}(i, o(g))=1 \\ 0 \leq i \leq o(g)}} g^{i} .
$$

We claim that $\gamma_{g} \in \mathcal{A}$, for all $g \in G$. Indeed, by Lemma $\amalg$.3. for each $\psi \in \mathcal{L} \operatorname{Aut}(G)$, there exists $r \in \mathbb{N}$ such that $\operatorname{gcd}(r,|G|)=1$ and $\psi(h)=h^{r}$, for all $h \in G$. Hence

$$
\psi\left(\gamma_{g}\right)=\sum_{\substack{\operatorname{gcd}(i, o(g))=1 \\ 0 \leq i \leq o(g)}} g^{i r}=\gamma_{g} .
$$


It is easy to see that given two elements $g_{1}, g_{2} \in G$, either $\gamma_{g_{1}}=\gamma_{g_{2}}$ or they have disjoint supports in $\mathbb{F} G$.

Let $\Gamma=\left\{g_{1}, g_{2}, \ldots, g_{s}\right\}$ be a complete set of elements in $G$ such that $\gamma_{g_{i}} \neq \gamma_{g_{j}}$, for $i \neq j$.

We claim that $\left\{\gamma_{g} \mid g \in \Gamma\right\}$ is an $\mathbb{F}$-basis of $\mathcal{A}$. Indeed, by considering the respective supports, it is clear that the set $\left\{\gamma_{g} \mid g \in \Gamma\right\}$ is linearly independent. Now given $\alpha \in \mathcal{A}$ and $g \in G$, the coefficients of $g$ and $g^{r}$ in $\alpha$, for all $r \in \mathbb{N}$ with $\operatorname{gcd}(r, o(g))=1$, must all be equal, by Lemma III.2. Thus, we may write:

$$
\alpha=a_{g_{1}} \gamma_{g_{1}}+a_{g_{2}} \gamma_{g_{2}}+\cdots+a_{g_{s}} \gamma_{g_{s}},
$$

hence $\left\{\gamma_{g} \mid g \in \Gamma\right\}$ is a basis for $\mathcal{A}$.

Notice that there exists a bijection between the set of cyclic subgroups of $G$ and the set $\left\{\gamma_{g} \mid g \in \Gamma\right\}$. By Theorem $\amalg .13$ there exists also a bijection between the set of the cyclic subgroups of $G$ and $\mathcal{S}_{c c}(G)$, hence $\operatorname{dim} \mathcal{A}=\left|\mathcal{S}_{c c}(G)\right|$.

By Lemma II.7 it is clear that $\mathcal{B} \subset \mathcal{A}$. In order to prove that $\mathcal{B}$ is linearly independent in $\mathbb{F} G$, assume that

$$
\sum_{K \in \mathcal{S}_{c c}(G)} a_{K} e_{K}=0,
$$

with $a_{K} \in \mathbb{F}$. Multiplying (8) by $e_{H}$, for a fixed $H \in \mathcal{S}_{c c}(G)$, we get $a_{H} e_{H}=0$, implying $a_{H}=0$, for all $H \in \mathcal{S}_{c c}(G)$. Therefore, $\mathcal{B}$ is linearly independent in $\mathbb{F} G$ and thus a basis for $\mathcal{A}$.

For an element $e_{H} \in \mathcal{B}$, assume, by way of contradiction, that $e_{H}=e_{1}+e_{2}$, with $e_{1}$ and $e_{2}$ orthogonal idempotents of $\mathcal{A}$. As $\mathcal{B}$ is a basis for $\mathcal{A}$, we can write

$e_{i}=\sum_{j=1}^{n_{i}} b_{j i} e_{H_{j}}, \quad$ with $e_{H_{j}} \in \mathcal{B}, b_{i j} \in \mathbb{F}, 1 \leq j \leq n, i=1,2$.

By Proposition II.6 we have either $e_{H_{j_{0}}} e_{H}=e_{H}$ (when $\left.H_{j_{0}}=H\right)$ or $e_{H_{j}} e_{H}=0$, for $j \neq j_{0}$. Hence $e_{i} e_{H}=b_{j_{0}} e_{H}$, if $H_{j_{0}}=H$, for some $j_{0}$ or $e_{i} e_{H}=0$, otherwise, $i=1,2$. As $e_{H}=e_{H}\left(e_{1}+e_{2}\right)$, we have that either $e_{1}=0$ or $e_{2}=e_{H}$ or $e_{1}=e_{H}$ and $e_{2}=0$. Therefore, $e_{H}$ is primitive in $\mathcal{A}$.

To finish the proof, write $1=\sum_{H \in \mathcal{S}_{c c}(G)} a_{H} e_{H}$, with $a_{H} \in \mathbb{F}$. For $K \in \mathcal{S}_{c c}(G)$, multiply by $e_{K}$ and get $e_{K}=\sum_{H \in \mathcal{S}_{c c}(G)} a_{H} e_{K} e_{H}=a_{K} e_{K}$ implying $a_{K}=1$, for all $K \in \mathcal{S}_{c c}(G)$.

Remark III.5. Notice that Lemma III.4 shows that

$$
\mathcal{A}=\bigoplus_{e_{H} \in \mathcal{B}} \mathbb{F} \cdot e_{H}
$$

Proposition III.6. Let $G$ be a finite abelian group and $\mathbb{F}$ a field such that $\operatorname{char}(\mathbb{F}) \backslash|G|$. If $e_{1}, e_{2} \in \mathcal{P}(\mathbb{F} G)$ and $H_{e_{1}}=$ $H_{e_{2}}$, then there exists an automorphism $\psi \in \mathcal{L} \operatorname{Aut}(G)$ whose linear extension to $\mathbb{F} G$ maps $e_{1}$ to $e_{2}$.

Proof: Let $H \in \mathcal{S}_{c c}(G)$ be such that $e_{1} e_{H}=e_{1}$, that is, $H_{e_{1}}=H$. By Theorem II.10 have

$$
e_{H}=\sum_{H_{f}=H} f, \quad \text { where } f \in \mathcal{P}(\mathbb{F} G) .
$$

By Proposition III.1, for any $\psi \in \mathcal{L} \operatorname{Aut}(G)$, we have

$$
\psi\left(e_{1}\right) \psi\left(e_{H}\right)=\psi\left(e_{1}\right) e_{\psi(H)}=\psi\left(e_{1}\right) e_{H}=\psi\left(e_{1}\right) .
$$

Let

$$
e_{0}=\sum \psi\left(e_{1}\right)
$$

where the sum runs over all distinct images of $e_{1}$ by $\psi \in$ $\mathcal{L} \operatorname{Aut}(G)$. Then $e_{0}$ is an idempotent and $\varphi\left(e_{0}\right)=e_{0}$, for all $\varphi \in \mathcal{L} \operatorname{Aut}(G)$, that is, $e_{0} \in \mathcal{A}$. Hence, by Lemma III.4

$$
e_{0}=\sum_{e_{H} \in \mathcal{B}} a_{H} e_{H}
$$

By construction of $e_{0}$ and (10), we have $e_{0} e_{H}=e_{0}$ and $e_{0} e_{K}=0$, for all $K \in \mathcal{S}_{c c}(G)$ with $K \neq H$. As $e_{H}$ is a primitive idempotent of $\mathcal{A}$, it follows $e_{0}=e_{H}$.

Now comparing the expression of $e_{H}$ in (9) and (11), by the uniqueness of the sum of primitive idempotents, each $f$ in the sum (9) is such that $f=\psi\left(e_{1}\right)$, for some $\psi \in \mathcal{L} \operatorname{Aut}(G)$. As $e_{2}$ is one of the idempotents in (9), the result follows.

Proposition III.7. Let $G$ be a finite abelian group and $\mathbb{F} a$ field such that $\operatorname{char}(\mathbb{F}) \backslash|G|$. If $e_{1}, e_{2} \in \mathcal{P}(\mathbb{F} G)$ are such that $\psi\left(H_{e_{1}}\right)=H_{e_{2}}$, for some $\psi \in \operatorname{Aut}(G)$, then there exists an automorphism $\theta \in \operatorname{Aut}(G)$ whose linear extension to $\mathbb{F} G$ maps $e_{1}$ and $e_{2}$, i.e., the ideals of $\mathbb{F} G$ generated by $e_{1}$ and $e_{2}$ are G-equivalent.

Proof: Since $\psi\left(H_{e_{1}}\right)=H_{e_{2}}$, for $\psi \in \operatorname{Aut}(G)$, by Lemma I.7, we have

$$
\psi\left(e_{1}\right) e_{H_{e_{2}}}=\psi\left(e_{1}\right) \psi\left(e_{H_{e_{1}}}\right)=\psi\left(e e_{H_{e_{1}}}\right)=\psi\left(e_{1}\right) .
$$

Hence, by the uniqueness, we have $H_{\psi\left(e_{1}\right)}=H_{e_{2}}$. Now, by Proposition III.6 there exists a local automorphism $\delta \in$ $\mathcal{L} \operatorname{Aut}(G)$ such that $\delta\left(\psi\left(e_{1}\right)\right)=e_{2}$. Therefore, taking $\theta=$ $\delta \psi \in \operatorname{Aut}(G)$, the result follows.

\section{Applications to CODing Theory}

We denote by $C_{n}$ the cyclic group of order $n$. In the context of Coding Theory, the following results appear in [9].

Theorem A [9. Theorem 3.6] Let $G$ be a finite abelian group of odd order and exponent $n$ and denote by $\tau(n)$ the number of divisors of $n$. Then there exist precisely $\tau(n)$ non $G$-equivalent minimal abelian codes in $\mathbb{F}_{2} G$.

Theorem B [9, Theorem 3.9] Let $G$ be a finite abelian group of odd order. Then two minimal abelian codes in $\mathbb{F}_{2} G$ are $G$ equivalent if and only if they have the same weight distribution.

Unfortunately these statements are not correct. The errors arise from the assumption, implicit in the last paragraph of [9, p. 167], that if $e$ and $f$ are primitive idempotents of $\mathbb{F}_{2} C_{m}$ and $\mathbb{F}_{2} C_{n}$, respectively, then $e f$ is a primitive idempotent of $\mathbb{F}_{2}\left[C_{m} \times C_{n}\right]$.

We exhibit below counterexamples to both Theorems A and $\mathrm{B}$ that were first communicated in [4]. However, Theorem A does hold under certain restrictive hypotheses, as we show in the next section. 
Proposition IV.1. Let $p$ be an odd prime such that $\overline{2}$ generates $U\left(\mathbb{Z}_{p^{2}}\right)$ and $G=\langle a\rangle \times\langle b\rangle$ an abelian group, with $o(a)=p^{2}$ and $o(b)=p$. Then $\mathbb{F}_{2} G$ has four inequivalent minimal codes, namely, the ones generated by the idempotents $e_{0}=\widehat{G}, e_{1}=\widehat{b}-\left\langle a^{p}\right\rangle \times\langle b\rangle, e_{2}=\widehat{a}-\widehat{G}$ and $e_{3}=\left\langle a^{p\rangle \times}\langle b\rangle-\widehat{G}\right.$.

Also all minimal codes of $\mathbb{F}_{2} G$ are described in the following table with their dimension and weight.

\begin{tabular}{|l|c|c|c|}
\hline Code & Primitive Idempotent & Dimension & Weight \\
\hline \hline$I_{0}$ & $e_{0}=\widehat{a} \widehat{b}=\widehat{G}$ & 1 & $p^{3}$ \\
\hline$I_{1}$ & $e_{1}=\widehat{b}-\left\langle a^{p}\right\rangle \times\langle b\rangle$ & $p^{2}-p$ & $2 p$ \\
\hline$I_{1 j}$ & $\begin{array}{c}e_{1 j}=\widehat{a^{j p} b}-\left\langle a^{\widehat{p}\rangle \times\langle}\langle b\rangle\right. \\
j=1, \ldots, p-1\end{array}$ & $p^{2}-p$ & $2 p$ \\
\hline$I_{2}$ & $e_{2}=\widehat{a}-\widehat{G}$ & $p-1$ & $2 p^{2}$ \\
\hline$I_{2 i}$ & $\begin{array}{c}e_{2 i}=\widehat{a b^{i}}-\widehat{G} \\
i=1, \ldots, p-1\end{array}$ & $p-1$ & $2 p^{2}$ \\
\hline$I_{3}$ & $e_{3}=\left\langle a^{p}\right\rangle \times\langle b\rangle-\widehat{G}$ & $p-1$ & $2 p^{2}$ \\
\hline \hline
\end{tabular}

Proof: In order to use Theorem II.3, first we need to find all subgroups $H$ of $G$ such that $G / H$ is cyclic. Notice that the $p+1$ distinct subgroups of order $p^{2}$ of $G$ are $\left\langle a b^{i}\right\rangle$, for $i=0, \ldots, p-1$, and $\left\langle a^{p}\right\rangle \times\langle b\rangle$. The $p+1$ distinct subgroups of order $p$ of $G$ are $\left\langle a^{j p} b\right\rangle$, for $j=0, \ldots, p-1$, and $\left\langle a^{p}\right\rangle$. The subgroups $\left\langle a^{j p} b\right\rangle$, for all $j=0, \ldots, p-1$, are contained only in $\left\langle a^{p}\right\rangle \times\langle b\rangle$ and $\left\langle a^{p}\right\rangle$ is contained in all subgroups of order $p^{2}$. Besides, all quotients of $G$ by these subgroups are cyclic, except $G /\left\langle a^{p}\right\rangle$ which is the unique noncyclic quotient of $G$. The quotient of $G$ by $\left\langle a^{p}\right\rangle \times\langle b\rangle$ is also cyclic.

Now applying Theorem $\amalg .3$, we have the following minimal codes generated by primitive idempotents.

The code $I_{0}=\mathbb{F}_{2} G \cdot e_{0}$, where $e_{0}=\widehat{G}$ and $\operatorname{dim} I_{0}=1$.

As $\langle b\rangle$ is uniquely contained in $\left\langle a^{p}\right\rangle \times\langle b\rangle$, we have $I_{1}=\mathbb{F}_{2} G \cdot e_{1}$, where $e_{1}=\widehat{b}-\left\langle a^{p\rangle \times}\langle b\rangle\right.$, and $\operatorname{dim} I_{1}=$ $\phi\left(p^{2}\right)=p^{2}-p$. The codes $I_{1 j}=\mathbb{F}_{2} G \cdot e_{1 j}$, where $e_{1 j}=\widehat{a^{j p} b}-\left\langle a^{p}\right\rangle \times\langle b\rangle$, for all $j=1, \ldots, p-1$, are all equivalent to $I_{1}$, since the extension to the group algebra $\mathbb{F}_{2} G$ of the isomorphism $\psi_{j}: G \rightarrow G$ given by $\psi(a)=a$ and $\psi(b)=a^{j p} b$, for each $j$, maps $I_{1}$ onto $I_{1 j}$.

Let $I_{2}=\mathbb{F}_{2} G \cdot e_{2}$, where $e_{2}=\widehat{a}-\widehat{G}$, and $I_{3}=\mathbb{F}_{2} G \cdot e_{3}$, where $e_{3}=\left\langle a^{p}\right\rangle \times\langle b\rangle-\widehat{G}$. We have $\operatorname{dim} I_{3}=\operatorname{dim} I_{2}=$ $\phi(p)=p-1$. We also have the codes $I_{2 i}=\mathbb{F}_{2} G \cdot e_{2 i}$, where $e_{2 i}=\widehat{a b^{i}}-\widehat{G}$, for $i=1, \ldots, p-1$, all equivalent to $I_{2}$ with corresponding isomorphism $\varphi_{i}: G \rightarrow G$ given by $\varphi(a)=a b^{i}$ and $\varphi(b)=b$.

We prove now that the codes $I_{k}$, with $k=0,1,2,3$, are four inequivalent minimal codes in $\mathbb{F}_{2} G$.

It is obvious that $I_{0}$ is not equivalent to any of the other codes $I_{k}$, for $k \neq 0$, and also that $I_{1}$ is not equivalent to either $I_{2}$ or $I_{3}$. Let us prove that $I_{2}$ and $I_{3}$ are inequivalent.

Notice that $\operatorname{supp}\left(e_{2}\right)=G \backslash\langle a\rangle$, which contains elements of order $p$, and $\operatorname{supp}\left(e_{3}\right)=G \backslash\left\langle a^{p}\right\rangle \times\langle b\rangle$, which only contains elements of order $p^{2}$. Hence, if there is an isomorphism $\psi: G \rightarrow G$ such that $\psi\left(e_{2}\right)=e_{3}$, we would have elements of order $p$ being mapped to elements of order $p^{2}$, a contradiction. Therefore, $I_{2}$ is not equivalent to $I_{3}$.

It is clear that the minimal code $I_{0}$ has weight $p^{3}$, as all its nonzero elements have this weight.

For $1 \leq j \neq k \leq p-1$, as $\operatorname{supp}\left(a^{j p \widehat{b}}\right) \cap \operatorname{supp}\left(a^{k p \widehat{b}}\right)=\emptyset$, the element $\left(a^{j p}+a^{k p}\right) e_{1}=\left(a^{j p}+a^{k p}\right) \widehat{b}$ is in $I_{1}$ and has weight $2 p$. Notice that $I_{1} \subset \mathbb{F}_{2} G \cdot \widehat{b}$, thus the weight of any element of $I_{1}$ must be a multiple of $p$. Hence, if there is an element in $I_{1}$ of weight $p$, it should be of the form $a^{i} \widehat{b}$. But $a^{i} \widehat{b} \cdot e_{1}=a^{i}\left(a^{p}+a 2 p+\cdots+a^{(p-1) p}\right) \widehat{b} \neq a^{i} \widehat{b}$ which implies $a^{i} \widehat{b} \notin I_{1}$, for any $1 \leq i$. Therefore, the weight of $I_{1}$ is $2 p$.

The weights of $I_{2}$ and $I_{3}$ will follow from the proof of the next proposition.

Proposition IV.2. The (inequivalent) minimal codes $I_{2}$ and $I_{3}$ of Proposition IV.1 have the same weight distribution.

Proof: An $\mathbb{F}_{2}$-basis for the code $I_{2}$ is

$$
\beta=\left\{\mu_{i}=(\hat{G}-\widehat{a}) b^{i}=\hat{G}-\hat{a} b^{i} \mid 1 \leq i \leq p-1\right\} .
$$

For $1 \leq i \neq j \leq p-1$, we have $\operatorname{supp}\left(\hat{a} b^{i}\right) \cap \operatorname{supp}\left(\hat{a} b^{j}\right)=\emptyset$. Hence, for an element $\alpha \in I_{2}$, we have:

Case 1. $\alpha$ is a sum of an even number of $\mu_{i}$ 's. Thus

$$
\alpha=\mu_{i_{1}}+\cdots+\mu_{i_{2 k}}=2 k \widehat{G}-\hat{a}\left(b^{i_{1}}+\cdots+b^{i_{2 k}}\right),
$$

which is an element of weight $2 k p^{2}$. Besides, in $I_{2}$ we have at least $\left(\begin{array}{c}p-1 \\ 2 k\end{array}\right)$ distinct elements with weight $2 k p^{2}$.

Case 2. $\alpha$ is a sum of an odd number of $\mu_{i}$ 's. Thus, for $k^{\prime} \geq 1$,

$$
\alpha=\mu_{i_{1}}+\cdots+\mu_{i_{2 k^{\prime}-1}}=\hat{G}-\hat{a}\left(b^{i_{1}}+\cdots+b^{i_{2 k^{\prime}-1}}\right),
$$

which is an element of weight

$$
p^{3}-\left(2 k^{\prime}-1\right) p^{2}=p^{2}\left(p-2 k^{\prime}+1\right)=2 k p^{2}
$$

(where $k^{\prime}=\frac{p+1-2 k}{2}$ ). Hence, in $I_{2}$, there are $\left(\begin{array}{c}p-1 \\ 2 k^{\prime}-1\end{array}\right)=$ $\left(\begin{array}{c}p-1 \\ p-2 k\end{array}\right)$ distinct elements with weight $2 k p^{2}$.

Therefore, for each $k \geq 1$, there are $\left(\begin{array}{c}p-1 \\ 2 k\end{array}\right)+$ $\left(\begin{array}{c}p-1 \\ p-2 k\end{array}\right)=\left(\begin{array}{c}p \\ 2 k\end{array}\right)$ elements of weight $2 k p^{2}$ in $I_{2}$.

Similarly, an $\mathbb{F}_{2}$-basis for the code $I_{3}$ is

$$
\gamma=\left\{\delta_{i}=e_{3} a^{i}=\widehat{G}-\widehat{a^{p}} \widehat{b} a^{i} \mid 1 \leq i \leq p-1\right\} .
$$

For $1 \leq i \neq j \leq p-1, \operatorname{supp}\left(\widehat{a^{p}} \widehat{b} a^{i}\right) \cap \operatorname{supp}\left(\widehat{a^{p}} \widehat{b} a^{j}\right)=\emptyset$. Hence, for an element $\alpha \in I_{3}$, we have:

Case 1. $\alpha$ is a sum of an even number of $\delta_{i}$ 's. Thus

$$
\alpha=\delta_{i_{1}}+\cdots+\delta_{i_{2 k}}=2 k \hat{G}-\widehat{a^{p}} \widehat{b}\left(a^{i_{1}}+\cdots+a^{i_{2 k}}\right),
$$

which is an element of weight $2 k p^{2}$. Besides, in $I_{3}$ we have at least $\left(\begin{array}{c}p-1 \\ 2 k\end{array}\right)$ distinct such elements with weight $2 k p^{2}$. 
Case 2. $\alpha$ is a sum of an odd number of $\delta_{i}$ 's. Thus, for $k^{\prime} \geq 1$,

$$
\alpha=\delta i_{1}+\cdots+\delta_{i_{2 k^{\prime}-1}}=\widehat{G}-\widehat{a^{p}} \widehat{b}\left(a^{i_{1}}+\cdots+a^{i_{2 k^{\prime}-1}}\right),
$$

which is an element of weight

$$
p^{3}-\left(2 k^{\prime}-1\right) p^{2}=p^{2}\left(p-2 k^{\prime}+1\right)=2 k p^{2},
$$

where $k^{\prime}=\frac{p+1-2 k}{2}$. Hence, in $I_{3}$, there are $\left(\begin{array}{c}p-1 \\ 2 k^{\prime}-1\end{array}\right)=$ $\left(\begin{array}{c}p-1 \\ p-2 k\end{array}\right)$ distinct elements with weight $2 k p^{2}$.

Therefore, also in $I_{3}$, for each $k \geq 1$, there are $\left(\begin{array}{c}p-1 \\ 2 k\end{array}\right)+$ $\left(\begin{array}{c}p-1 \\ p-2 k\end{array}\right)=\left(\begin{array}{c}p \\ 2 k\end{array}\right)$ elements of weight $2 k p^{2}$.

As $\sum_{k=1}^{(p-1) / 2}\left(\begin{array}{c}p \\ 2 k\end{array}\right)=2^{p-1}$, this proves that the weight distribution of $I_{2}$ and $I_{3}$ are the same, but $I_{2}$ and $I_{3}$ are not equivalent. Besides, the weight of these codes is $2 p^{2}$.

Observe that the group $G$ of Proposition IV.1 has exponent $p^{2}$ and $\tau\left(p^{2}\right)=3$, however, $\mathbb{F}_{2} G$ has four inequivalent minimal codes. This is a counterexample to Theorem A.

Notice that Proposition IV.2 actually exhibits a counterexample also to Theorem B.

In the following proposition, we study the minimal codes in $\mathbb{F}_{2}\left(C_{p^{n}} \times C_{p}\right)$, for an odd prime $p$ and $n \geq 3$. Its proof is similar to the proof of Proposition IV.1 This gives a whole family of counterexamples to Theorem A.

Proposition IV.3. Let $n \geq 3$ be a positive integer and $p$ an odd prime such that $\overline{2}$ generates $U\left(\mathbb{Z}_{p^{2}}\right)$ and $G=\langle a\rangle \times\langle b\rangle$ be an abelian group, with $o(a)=p^{n}$ and $o(b)=p$. Then the

\begin{tabular}{|c|c|c|}
\hline Code & Dimension & Weight \\
\hline$\overline{I_{0}}=\langle\widehat{a} \widehat{b}\rangle=\langle\widehat{G}\rangle$ & 1 & $p^{n+1}$ \\
\hline$I_{1}=\left\langle\left\langle a^{\widehat{p}\rangle \times\langle b\rangle-\widehat{G}\rangle}\right.\right.$ & $p-1$ & $2 p^{n}$ \\
\hline $\begin{array}{l}I_{1 i}=\left\langle\widehat{a b^{i}}-\widehat{G}\right\rangle \\
i=0, \ldots, p-1\end{array}$ & $p-1$ & $2 p^{n}$ \\
\hline$I_{2}=\left\langle\left\langle a^{\left.p^{2}\right\rangle \times}\langle b\rangle-\left\langle a^{p}\right\rangle \times\langle b\rangle\right\rangle\right.$ & $p(p-1)$ & $2 p^{n-1}$ \\
\hline $\begin{array}{l}I_{2 i}=\left\langle\widehat{a^{p} b^{i}}-\left\langle\widehat{\left.a^{p}\right\rangle \times\langle b\rangle}\right\rangle\right. \\
i=1, \ldots, p-1\end{array}$ & $p(p-1)$ & $2 p^{n-1}$ \\
\hline$\ldots$ & $\ldots$ & \\
\hline$I_{k}=\left\langle\left\langle a^{p^{k}}\right\rangle \times\langle b\rangle-\left\langle a^{p^{k-1}}\right\rangle \times\langle b\rangle\right\rangle$ & $p^{k-1}(p-1)$ & $2 p^{n-k+1}$ \\
\hline $\begin{array}{l}I_{k i}=\left\langle a^{p^{k-1}} b^{i}-\left\langle a^{p^{k-1}}\right\rangle \times\langle b\rangle\right\rangle \\
i=1, \ldots, p-1\end{array}$ & $p^{k-1}(p-1)$ & $2 p^{n-k+1}$ \\
\hline$\ldots$ & $\ldots$ & \\
\hline$I_{n-1}=\left\langle\widehat{\langle b\rangle}-\left\langle a^{p^{n-2}}\right\rangle \times\langle b\rangle\right\rangle$ & $p^{(n-1)}(p-1)$ & $2 p$ \\
\hline $\begin{array}{l}I_{n-1, i}=\left\langle\widehat{a^{p^{n-1}}} b^{i}-\left\langle a^{p^{n-2}}\right\rangle \times\langle b\rangle\right\rangle \\
i=1, \ldots, p-1\end{array}$ & $p^{(n-1)}(p-1)$ & $2 p$ \\
\hline
\end{tabular}
minimal codes of $\mathbb{F}_{2} G$ are described in the following table.

There are $2 n$ inequivalent minimal codes in $\mathbb{F}_{2}\left(C_{p^{n}} \times C_{p}\right)$.

The following table presents the correspondence between the classes of $G$-isomorphisms of subgroups and the $G$ equivalence classes of minimal codes in $\mathbb{F}_{2}\left(C_{p^{n}} \times C_{p}\right)$, listing representatives of these classes.

\begin{tabular}{|c|c|}
\hline Subgroups & Codes \\
\hline \hline$G$ & $I_{0}=\langle\widehat{G}\rangle$ \\
\hline$\langle a\rangle$ & $I_{11}=\langle\widehat{a}-\widehat{G}\rangle$ \\
\hline$\left\langle a^{p}\right\rangle \times\langle b\rangle$ & $I_{1}=\left\langle\left\langle a^{p}\right\rangle \times\langle b\rangle-\widehat{G}\right\rangle$ \\
\hline$\left\langle a^{p} b\right\rangle$ & $I_{21}=\left\langle\widehat{a^{p} b}-\left\langle a^{\widehat{p}}\right\rangle \times\langle b\rangle\right\rangle$ \\
\hline$\left\langle a^{p^{2}}\right\rangle \times\langle b\rangle$ & $I_{2}=\left\langle\left\langle a^{p^{2}}\right\rangle \times\langle b\rangle-\left\langle a^{p}\right\rangle \times\langle b\rangle\right\rangle$ \\
\hline$\ldots$ & $\cdots$ \\
\hline$\left\langle a^{p^{k}} b\right\rangle$ & $I_{k+1,1}=\left\langle\widehat{a^{p^{k}}} b-\left\langle a^{p^{k}}\right\rangle \times\langle b\rangle\right\rangle$ \\
\hline$\left\langle a^{p^{k+1}}\right\rangle \times\langle b\rangle$ & $I_{k+1}=\left\langle\left\langle a^{p k+1}\right\rangle \times\langle b\rangle-\left\langle a^{p^{k}}\right\rangle \times\langle b\rangle\right\rangle$ \\
\hline$\ldots$ & $\ldots$ \\
\hline$\langle b\rangle$ & $I_{n-1}=\left\langle\widehat{b}-\left\langle a^{p^{n-1}}\right\rangle \times\langle b\rangle\right\rangle$ \\
\hline \hline
\end{tabular}

\section{A POSITIVE RESUlT FOR CODES}

In [4] we showed that Theorem A holds in the special case of minimal codes in $\mathbb{F}_{2}\left(C_{p^{n}} \times C_{p^{n}}\right)$. We now prove this Theorem in a more general situation.

Lemma V.1. If $H$ is a cyclic subgroup of order $p^{s}$ in a group $G \cong \underbrace{C_{p^{r}} \times \cdots \times C_{p^{r}}}$, with $s \leq r$, then there exists a cyclic subgroup of $\stackrel{m}{G}$, of order $p^{r}$, containing $H$.

Proof: Write $G=\left\langle g_{1}\right\rangle \times \cdots \times\left\langle g_{m}\right\rangle$, with $o\left(g_{i}\right)=p^{r}$, $1 \leq i \leq m$. Since $H$ is a cyclic subgroup of $G$, we have $H=$ $\left\langle g_{1}^{j_{1}} p^{t_{1}} g_{2}^{j_{2} p^{t_{2}}} \cdots g_{m}^{j_{m}} p^{t_{m}}\right\rangle$, with $\operatorname{gcd}\left(j_{i}, p\right)=1$ and $0 \leq t_{i} \leq r$, $1 \leq i \leq m$. Then one of the exponents $t_{k}, 1 \leq k \leq m$, is minimal and, for such $t_{k}$, we consider the element $a=$ $g_{1}^{j_{1} p^{t_{1}-t_{k}}} \cdots g_{k}^{j_{k} p^{0}} \cdots g_{m}^{j_{m} p^{t_{m}-t_{k}}} \in G$. As $\operatorname{gcd}\left(j_{k}, p\right)=1$, we have $o(a)=p^{r}$ and $\left\langle a^{p^{t_{k}}}\right\rangle=H$, as $G \cong \underbrace{C_{p^{r}} \times \cdots \times C_{p^{r}}}_{m}$.

Theorem V.2. Let $m$ and $r$ be positive integers. If $G=$ $\underbrace{C_{p^{r}} \times \cdots \times C_{p^{r}}}_{m}$ is a finite abelian p-group, then any cocyclic subgroup of $G$ contains a subgroup isomorphic to $C_{p^{r}} \times \cdots \times C_{p^{r}}$. Hence the subgroups of $G$ isomorphic to $\underbrace{}_{(m-1)}$ $\underbrace{}_{p^{r}} \times \cdots \times C_{p^{r}}$ are precisely the minimal co-cyclic sub$(m-1)$ groups of $G$.

Proof: Let $H \in \mathcal{S}_{c c}(G)$. Then, by Theorem II.13(3), $H^{\perp}$ is a cyclic subgroup of $G^{*}$. By Theorem II.13 1) and Lemma V.1, there exists $K^{\perp} \cong C_{p^{r}}$ a subgroup of $G^{*}$ such that $H^{\perp} \subset K^{\perp}$. Then $K=\Psi^{-1}\left(K^{\perp}\right)$ is a subgroup of $G$ such that $K \cong \underbrace{C_{p^{r}} \times \cdots \times C_{p^{r}}}$ and $K \subset H$, proving the theorem.

$$
(m-1)
$$

As a consequence of the results above, we get:

Proposition V.3. Let $m$ and $r$ be positive integers. If $G=$ $C_{p^{r}} \times \cdots \times C_{p^{r}}$ is a finite abelian p-group and $\mathbb{F}$ is a field with $\operatorname{char}(\mathbb{F}) \neq p$, then a primitive idempotent of $\mathbb{F} G$ is of the form $\widehat{K} \cdot e_{h}$, where $K$ is a subgroup of $G$ isomorphic to 
$\underbrace{C_{p^{r}} \times \cdots \times C_{p^{r}}}_{(m-1)}$ and $e_{h}$ is a primitive idempotent of $\mathbb{F}\langle h\rangle$, where $h \in G$ is such that $G=\langle h\rangle \times K$ and $\langle h\rangle \cong C_{p^{r}}$.

Proof: Let $e$ be a primitive idempotent of $\mathbb{F} G$ and $H$ be the unique co-cyclic subgroup of $G$ such that $e=\widehat{H} \cdot e$, by Theorem ??. By Theorem V.2 there exists $K \cong \underbrace{C_{p^{r}} \times \cdots \times C_{p^{r}}}$ contained in $H$ such that $e \widehat{K}=e$.

$(m-1)$

Let $h \in G$ be such that $G / K=\langle K h\rangle$. Since $|G / K|=p^{r}$ and $\exp (G)=p^{r}$, it follows directly that $o(h)=p^{r}$. Hence, we can write:

$$
\begin{aligned}
e & =\widehat{K} \cdot e \\
& =\widehat{K}\left[\left(\sum_{g \in K} \alpha_{0, g} g\right) 1+\cdots+\left(\sum_{g \in K} \alpha_{p^{r}-1, g} g\right) h^{p^{r}-1}\right] \\
& =\widehat{K}\left(\beta_{0} \cdot 1+\beta_{1} \cdot h+\cdots+\beta_{p^{r}-1} \cdot h^{p^{r}-1}\right)
\end{aligned}
$$

where $\beta_{i}=\sum_{g \in K} \alpha_{i, g} \in \mathbb{F}$ and $h^{t} \notin K, 1 \leq t \leq p^{r}-1$.

Let $\psi: G \longrightarrow G / K$ be the natural homomorphism. Since $e=\widehat{K} \cdot e$ is an idempotent in $\mathbb{F} G$, we have

$$
\begin{aligned}
\psi(e) & =\psi(\widehat{K} \cdot e) \\
& =\psi\left(\widehat{K}\left(\beta_{0} \cdot 1+\beta_{1} \cdot h+\cdots+\beta_{p^{r}-1} \cdot h^{p^{r}-1}\right)\right) \\
& =\beta_{0} \cdot 1+\beta_{1} \cdot h+\cdots+\beta_{p^{r}-1} \cdot h^{p^{r}-1}
\end{aligned}
$$

is also an idempotent.

Claim: $e_{h}=\psi(e)=\beta_{0} \cdot 1+\beta_{1} \cdot h+\cdots+\beta_{p^{r}-1} \cdot h^{p^{r}-1}$ is a primitive idempotent in $\mathbb{F}\langle h\rangle \cong \mathbb{F}(G / K)$.

Indeed, if $e_{h}=e_{1}+e_{2}$, with $e_{1}$ and $e_{2}$ orthogonal idempotents in $\mathbb{F}\langle h\rangle$, then

$$
e=\widehat{K} \cdot e_{h}=\widehat{K} \cdot e_{1}+\widehat{K} \cdot e_{2},
$$

with $\left(\widehat{K} \cdot e_{i}\right)^{2}=\widehat{K} \cdot e_{i}$, for $i=1,2$, and $\widehat{K} \cdot e_{1} \widehat{K} \cdot e_{2}=0$, as $e_{1} \cdot e_{2}=0$. Since $e$ is a primitive idempotent, then either $\widehat{K} \cdot e_{1}=0$ or $\widehat{K} \cdot e_{2}=0$. Suppose $\widehat{K} \cdot e_{1}=0$ and write $e_{1}=\gamma_{0} \cdot 1+\gamma_{1} \cdot h+\gamma_{2} \cdot h^{2}+\cdots+\gamma_{p^{r}-1} \cdot h^{p^{r}-1}$. Then

$$
\begin{aligned}
0 & =\widehat{K} \cdot e_{1} \\
& =\frac{1}{|K|}\left(\sum_{k \in K} k\right)\left(\gamma_{0}+\gamma_{1} \cdot h+\cdots+\gamma_{p^{r}-1} \cdot h^{p^{r}-1}\right) \\
& =\frac{1}{|K|}\left[\left(\sum_{k \in K} \gamma_{0} k\right)+\cdots+\left(\sum_{k \in K} \gamma_{p^{r}-1} k\right) h^{p^{r}-1}\right]
\end{aligned}
$$

implies $\gamma_{i}=0,0 \leq i \leq p^{r}-1$, as the summands in (13) have disjoint supports in $\mathbb{F} G$. This proves the proposition.

These results can be applied and extended as follows.

Corollary V.4. Let $m$ and $r$ be positive integers, a finite abelian p-group $G=\underbrace{C_{p^{r}} \times \cdots \times C_{p^{r}}}_{m}$ and $\mathbb{F}_{q}$ a finite field with $q$ elements such that $o(\bar{q})=\phi\left(p^{r}\right)$ in $U\left(\mathbb{Z}_{p^{r}}\right)$. Then the minimal abelian codes in $\mathbb{F}_{q} G$ are as follows:

\begin{tabular}{|c|c|c|}
\hline \hline Primitive Idempotent & Dimension & Weight \\
\hline \hline$\widehat{G}$ & 1 & $p^{r m}$ \\
\hline$\widehat{K}\left(\widehat{h^{p}}-\widehat{h}\right)$ & $p-1$ & $2 p^{r(m-1)+(r-1)}$ \\
\hline$\widehat{K}\left(\widehat{h^{p^{2}}}-\widehat{h^{p}}\right)$ & $p(p-1)$ & $2 p^{r(m-1)+(r-2)}$ \\
\hline$\widehat{K}\left(\widehat{h^{p^{3}}}-\widehat{h^{p^{2}}}\right)$ & $p^{2}(p-1)$ & $2 p^{r(m-1)-(r-3)}$ \\
\hline$\cdots$ & $\cdots$ & \\
\hline$\widehat{K}\left(\widehat{h^{p^{i}}}-\widehat{h^{p^{i-1}}}\right)$ & $p^{i-1}(p-1)$ & $2 p^{r(m-1)-(r-i)}$ \\
\hline$\cdots$ & $\cdots$ & \\
\hline$\widehat{K}\left(1-\widehat{h^{p^{r-1}}}\right)$ & $p^{r-1}(p-1)$ & $2 p^{r(m-1)}$ \\
\hline \hline
\end{tabular}

where $h$ is as in Proposition V.3 Consequently, the number of non $G$-equivalent minimal abelian codes is $r+1=\tau\left(p^{r}\right)$.

Corollary V.5. Let $n \geq 2$ be an integer, $G=\underbrace{C_{n} \times \cdots \times C_{n}}_{m}$ an abelian group and $\mathbb{F}_{q}$ a finite field such that $\operatorname{gcd}(q, n)=1$. Then the primitive idempotents of $\mathbb{F}_{q} G$ are of the form $\widehat{K} \cdot e_{h}$, where $K$ is a subgroup of $G$ isomorphic to $\underbrace{C_{n} \times \cdots \times C_{n}}_{(m-1)}$, $h \in G$ is such that $G=K \times\langle h\rangle$ and $e_{h}$ is a primitive idempotent of $\mathbb{F}_{q}\langle h\rangle$.

Proof: Let $n=p_{1}^{n_{1}} p_{2}^{n_{2}} \cdots p_{t}^{n_{t}}$, with $p_{i}$ rational primes, $1 \leq i \leq t$, and $p_{i} \neq p_{j}$, for $i \neq j$ and $n_{i} \geq 1$. Let $G_{i}=$ $\underbrace{C_{p_{i}^{r_{i}}} \times \cdots \times C_{p_{i}^{r_{i}}}}$ be the $p_{i}$-Sylow subgroup of $G$.

Let $0 \stackrel{m}{\neq} e \in \mathbb{F}_{q} G$ be a primitive idempotent. By Lemma there exists a unique $H \in \mathcal{S}_{c c}(G)$ such that $e \cdot e_{H}=e$ and $e \cdot e_{K}=0$, for any other $K \in \mathcal{S}_{c c}(G)$, with $K \neq H$. By (2), $e_{H}=e_{H_{1}} e_{H_{2}} \cdots e_{H_{t}}$, where $e_{H_{i}}=\widehat{G_{i}}$ or $e_{H_{i}}=\widehat{H_{i}}-\widehat{H_{i}^{\sharp}}$, for $H_{i}$ the $p_{i}$-Sylow of $H$. Hence, as $0 \neq e$ is primitive, we have

$$
e \cdot e_{H}=e \quad \Rightarrow \quad e \cdot e_{H_{i}}=e
$$

By Proposition ?? and Theorem V.2, there exists $K_{i}=$ $C_{p_{i}^{r_{i}}} \times \cdots \times C_{p_{i}^{r_{i}}}$ a subgroup of $G_{i}$ such that $K_{i} \leq H_{i}$ and $(m-1)$

$e=e \cdot \widehat{K}_{i}$, for all $1 \leq i \leq t$. Hence

$$
\begin{aligned}
& e=e \cdot \widehat{K_{1}}=e \cdot \widehat{K_{2}} \widehat{K_{1}} \\
& \text {. } \\
& =e \cdot \widehat{K_{t}} \cdots \widehat{K_{2}} \widehat{K_{1}}=e K_{t} \widehat{\cdots K_{2}} K_{1} \\
& \cong e(\underbrace{C_{n} \times \cdots \times C_{n}}_{(m+1) \text { factors }}) \text {. } \\
& (m-1) \text { factors }
\end{aligned}
$$

Let $K=\underbrace{C_{n} \times \cdots \times C_{n}}_{(m-1) \text { factors }}$. Then $G / K \cong C_{n}$. Take $a \in G$ such that $G / K=\langle K a\rangle$. Similarly to the proof of Theorem V.3, we show that $o(a)=n$ and that $\psi(e)$ is a primitive idempotent of $\mathbb{F}_{q}\langle a\rangle$, where $\psi: \mathbb{F}_{q} G \longrightarrow \mathbb{F}_{q}(G / K)$.

Using the results from this section and from Section III, we obtain the following.

Theorem V.6. Let $G$ be a finite abelian group of exponent $n$ and $\mathbb{F}$ a finite field such that char $(\mathbb{F}) \backslash|G|$. Then the number 
of non G-equivalent minimal abelian codes is precisely $\tau(n)$ if and only if $G$ is a direct product of cyclic groups isomorphic to one another.

\section{REFERENCES}

[1] S.D. Berman, Semisimple cyclic and abelian codes, II, Kybernetika 3 (1967) 21-30.

[2] O. Broche and A. del Río, Wedderburn decomposition of finite group algebras, Finite Fields and their Applications 13 (2007) 71-79.

[3] R.A. Ferraz and C. Polcino Milies, Idempotents in group algebras and minimal abelian codes, Finite Fields and their Applications 13 (2007) 382-393.

[4] R.A. Ferraz, M. Guerreiro and C. Polcino Milies, Minimal codes in binary abelian group algebras, Information Theory Workshop (ITW) IEEE (2011) 225-228. (Digital Object Identifier: 10.1109/ITW.2011.6089383)

[5] E.G. Goodaire, E. Jespers and C. Polcino Milies, Alternative Loop Rings, North-Holland Mathematics Studies 184, Amsterdam, Holland: Elsevier, 1996.

[6] C.J. Hillar and D.L. Rhea, Automorphisms of finite abelian groups, American Math. Monthly 114 N. 10 (2007) 917-923.

[7] E. Jespers, G. Leal and A. Paques, Central idempotents in the rational group algebra of a finite nilpotent group, Journal of Algebra and its Applications 2 No. 1 (2003) 57-62.

[8] F.J. MacWilliams,Binary codes which are ideals in the group algebra of an abelian group, Bell System Tech. Journal 44 (1970) 987-1011.

[9] R.L. Miller, Minimal codes in abelian group algebras, Journal of Combinatorial Theory, Series A 26 (1979) 166-178.

[10] A. Olivieri, A. del Río and J.J. Simón, On monomial characters and central idempotents of rational group algebras, Comm. Algebra 32 (4) (2004) 1531-1550.

[11] C. Polcino Milies and S.K. Sehgal, An Introduction to Group Rings, Kluwer Academic Publishers, Dordrecht???, 2002.

[12] J.J. Rotman, An Introduction to the Theory of Groups, 4th Ed., SpringerVerlag, New York, 1995. 